\title{
新型金属胶束用作水解金属模拟酶 *
}

\author{
戴秋云 黄启斌 邓云度
}

(北京药物化学研究所, 北京 102205)

\section{关键词 金属胶束 水解金属模拟酶 羖酸酯 催化水解}

阳离子表面活性剂型金属胶束 (metallomicelles) 是近期出现的一种新型功能胶束 ${ }^{[1,2]}$, 由 阳离子表面活性剂功能络合物自身或在共表面活性剂存在下形成, 它不仅水溶性好, 且具有双 层正电荷结构, 是模拟水解金属酶配位、疏水、静电作用方式的优良体系.

羟基及碱性基团是天然水解酶中重要的催化功能基, 在合成分子中有效构建这些功能基 使之具有类似天然酶的催化活性,是水解模拟酶设计的最主要问题. 目前非金属模拟酶中难 以使羟基解离成为有效的亲核试剂, 但在一些设计巧妙的水解金属模拟酶中 ${ }^{[3,4]}$, 金属离子的 正电荷可加速差基解离, 使差基在中性条件下成为有效的亲核试剂.

为研究阳离子表面活性剂型金属胶束中羟基的活化特征, 我们合成了 4 种新的阳离子及 非离子型长链吡啶与二级羟基功能配体 $(1 \mathrm{a} \sim 1 \mathrm{c}, 2)$, 测定了其在铜锌离子存在下对羧酸酯 PNPP 的催化水解动力学, 结果表明: 差基的活化受几何构型及金属离子性质控制, 靠近功能 基的季铵正电荷对络合物的形成及活性存在显著影响.

\section{1 实验}

\section{1 材料}

长链配体 $1 \mathrm{a} \sim 1 \mathrm{c}$ 由 $\omega$-溴代吡啶乙酮与三级胺的加成产物氢化而得, 2 由氡基吡啶与格氏 试剂反应产物氢化得到, 结构均经元素分析、红外、核磁共振、质谱证实. 底物 PNPP 按文献 [5]合成, 动力学测试用试剂均为分析纯.<smiles>C[N+](C)(CBr)CC(O)c1ccccn1</smiles>

$n=12 \quad 1 \mathrm{a}$

$n=14 \mathrm{lb}$ $n=18 \mathrm{Ic}$

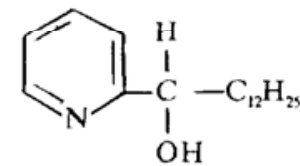

2<smiles></smiles>

PNPP

\section{2 动力学方法}

慢反应用 Shimadzu-265 紫外分光光度计监测产物对硝基苯酚的形成, 快反应使用 APL 公司的 SF. $17 \mathrm{MV}$ 停流仪监测, 监测波长为 $400 \mathrm{~nm}$.

1996-01-22 收稿, 1996-05-07 收修改稿

* 国家自然科学基金资助项目 


\section{2 结果与讨论}

\section{1 锌铜离子活化羟基的特征}

图 1, 2 为不同锌铜离子浓度下 $1 \mathrm{a} \sim 1 \mathrm{c}$ 及 2 对 PNPP 的反应活性. 从图 1 可看出, 随着锌 离子浓度的增加, 配体的反应活性迅速提高, 当锌离子浓度超过配体浓度时, 反应速率增加缓 慢, 反应活性顺序为 $1 \mathrm{c} \mathrm{Zn}_{\mathrm{n}}>1 \mathrm{bZn}>1 \mathrm{aZn}$. 而对于铜离子胶束(图 2), 虽然配体的反应活性亦随 着铜离子浓度的增加而增加, 但当铜离子浓度为配体浓度一半时, $2 \mathrm{Cu}$ 的反应活性达最大值, 而 $1 \mathrm{a} \sim 1 \mathrm{c}$ 反应活性很小, 且 $1 \mathrm{aCu}>1 \mathrm{bCu} \approx 1 \mathrm{cCu}$, 与锌离子胶束显著不同. 这表明反应活性与 锌铜离子形成络合物的能力有关, 锌离子一般易形成四面体型络合物, 铜离子易形成平面或畸 形八面络合物, 铜离子比锌离子更易形成多配体络合物. $1 \mathrm{a} \sim 1 \mathrm{c}$ 立体阻碍大, 与铜离子形成 多配体络合物后, 不易与底物 PNPP 结合, 活性较低, 且随疏水性 (烷基链) 增加, 形成多配体络 合物的能力愈强.

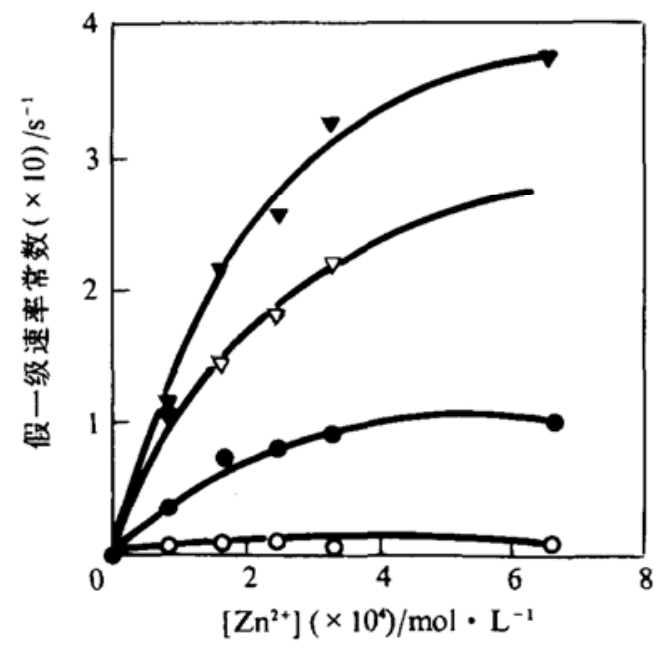

图 1 锌离子对反应的影响

$[\mathrm{L}]=3.3 \times 10^{-4} \mathrm{~mol} / \mathrm{L},[\mathrm{PNPP}]=3.3 \times 10^{-5} \mathrm{~mol} / \mathrm{L}$, $[\mathrm{CTAB}]=2.5 \times 10^{-3} \mathrm{~mol} / \mathrm{L}, \mathrm{pH}=7.76,[\mathrm{KCl}]=0.05$ $\mathrm{mol} / \mathrm{L}, 0.05 \mathrm{~mol} / \mathrm{L} \mathrm{N}$-乙基吗啡啉, 乙醇 $9 \%, T=25 \mathrm{C}$

○示 2, ○示 $1 a, \nabla$ 示 $1 b, \nabla$ 示 $1 c$

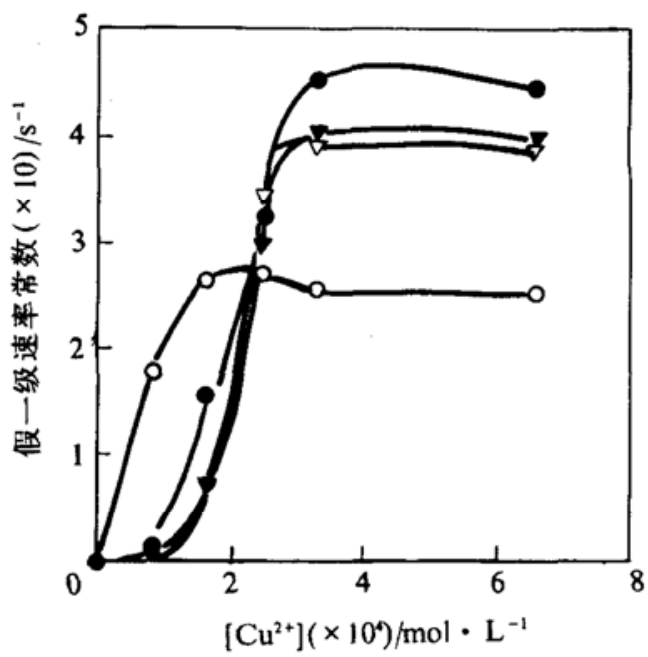

图 2 铜离子对反应的影响 $[\mathrm{L}]=3.3 \times 10^{-4} \mathrm{~mol} / \mathrm{L},[\mathrm{PNPP}]=3.3 \times 10^{-5} \mathrm{~mol} / \mathrm{L}$, $[\mathrm{CTAB}]=2.5 \times 10^{-3} \mathrm{~mol} / \mathrm{L}, \mathrm{pH}=7.11,[\mathrm{KCl}]=0.05$ $\mathrm{mol} / \mathrm{L}, 0.05 \mathrm{~mol} / \mathrm{L} \mathrm{N}$-乙基吗啡啉, 乙醇 $9 \%, T=25{ }^{\circ} \mathrm{C}$

○示 2, 示 $1 a, \nabla$ 示 $1 b, \nabla$ 示 $1 c$

\section{2 季铵头的影响}

实验表明 $1 \mathrm{a} \sim 1 \mathrm{c}$ 铜离子胶束在 $\mathrm{pH}>7.11$ 后活性增加幅度很小, 在 $\mathrm{pH}=7.76$ 时, $1 \mathrm{a} \sim 1 \mathrm{c}$ 锌离子胶束的反应活性与其铜离子胶束相当, 这种现象是首次观察到, 在目前报道的羟基胶束 中均是铜离子胶束的反应活性显著高于锌离子胶束. 我们认为该现象与上述络合物的构型有 关外, 还与季铵头的影响有关. 一般铜离子比锌离子更能加速羟基离解, 铜离子胶束在低 $\mathrm{pH}$ 下活性高, 同时铜离子的正电荷对羟基的亲核性降低也较大, 在较高 $\mathrm{pH}$ 下, 季铵头的吸电子 效应可进一步减弱羟基的亲核性. 而锌离子对羟基的亲核性降低较少, 在低 $\mathrm{pH}$ 下羟基难以 解离 (可从 $\mathrm{pH}$ 与 $1 \mathrm{cZn}$ 的速率常数的关系看出, 未列出), $\mathrm{pH}$ 较高时羟基因季铵头的作用变得 易于解离. 
季䯃头的存在也导致共胶束的影响显著不同. 实验观察到 $1 \mathrm{bZn}$ 在 CTAB, TritonX-100 共胶束临界胶束浓度处反应速率最大 (CTAB, SDS, TritonX-100 的 CMC 分别为 $9.2 \times 10^{-4}$; $\left.8.1 \times 10^{-3}, 3.0 \times 10^{-4} \mathrm{~mol} / \mathrm{L}^{[6]}\right)$, 而在 SDS 中, 反应速率在大大低于 SDS 的 CMC 处 $(2 \times$ $10^{-3} \mathrm{~mol} / \mathrm{L}$ ) 最大, 且 SDS 中的反应活性较在 CTAB 及 TritonX-100 中大 $3 \sim 4$ 倍. $1 \mathrm{bZn}$ 不在 SDS 的 CMC 处活性最强, 可能与 SDS 阴离子头与 $1 \mathrm{bZn}$ 季铵头静电作用有关, 静电作用导致 临界胶束浓度降低, 胶束疏水性增强, 活性增加.

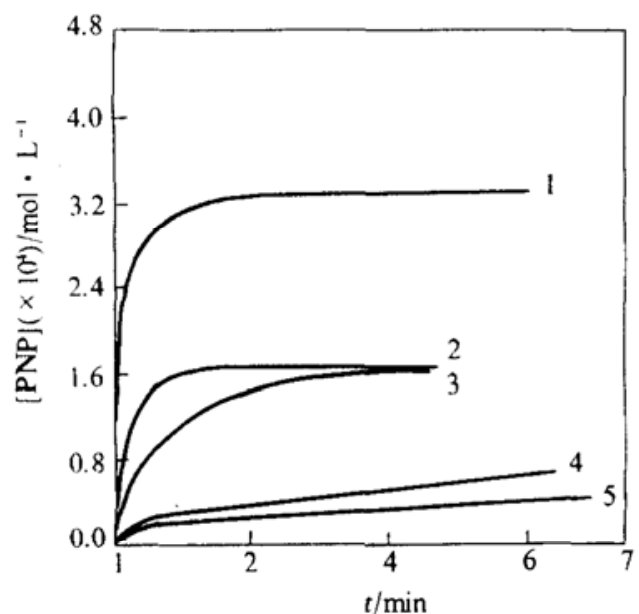

图 $31 \mathrm{bCu}$ 及 $1 \mathrm{bZn}$ 对 PNPP 水解的 burst 动力学 $[1 \mathrm{~b}]=3.3 \times 10^{-5} \mathrm{~mol} / \mathrm{L},[\mathrm{CTAB}]=2.5 \times 10^{-3} \mathrm{~mol} / \mathrm{L}$, $\mathrm{pH}=7.11(1 \mathrm{bCu}), \mathrm{pH}=7.76(1 \mathrm{bZn}), T=25^{\circ} \mathrm{C}$ $1-\left[\mathrm{Cu}^{2+}\right]=1.65 \times 10^{-4} \mathrm{~mol} / \mathrm{L},[\mathrm{PNPP}]=3.3 \times 10^{-4}$ $\mathrm{mol} / \mathrm{L} ; 2-\left[\mathrm{Cu}^{2+}\right]=1.65 \times 10^{-4} \mathrm{~mol} / \mathrm{L},[\mathrm{PNPP}]=1.65$ $\times 10^{-4} \mathrm{~mol} / \mathrm{L} ; 3-\left[\mathrm{Cu}^{2+}\right]=6.6 \times 10^{-5} \mathrm{~mol} / \mathrm{L},[\mathrm{PNPP}]$ $=1.65 \times 10^{-4} \mathrm{~mol} / \mathrm{L} ; 4-\left[\mathrm{Zn}^{2+}\right]=1.65 \times 10^{-4} \mathrm{~mol} / \mathrm{L}$, $[\mathrm{PNPP}]=1.65 \times 10^{-4} \mathrm{~mol} / \mathrm{L} ; 5-\left[\mathrm{Zn}^{2+}\right]=3.3 \times 10^{-5}$ $\mathrm{mol} / \mathrm{L},[\mathrm{PNPP}]=1.65 \times 10^{-4} \mathrm{~mol} / \mathrm{L}$

\section{3 催化效率与机制}

当 $[1 \mathrm{c}]=\left[\mathrm{Zn}^{2+}\right]=3.3 \times 10^{-4} \mathrm{~mol} / \mathrm{L}$ 时 $($ 条 件如图 1), 对 PNPP 反应的假一级速率常数达 $0.33 \mathrm{~s}^{-1}$, 二级速率常数为 $1.0 \times 10^{3} \mathrm{~L} / \mathrm{mol}$. $\mathrm{s}^{-1}$, 比自发水解增加 $10^{3}$ 倍, 其活性高于活性 最高的小分子锌络合物 10 倍以上 ${ }^{[3]}$, 也高于单 咪唑、单羟基锌离子胶束及吡啶、酰胺、一级羟 基功能基的锌离子胶束 ${ }^{[3,7]}$ 的活性, 与锌离子 胶束中活性最高的含双咪唑及羟基胶束相 当 ${ }^{[3]}$.

实验观察到锌铜离子胶束对 PNPP 水解是 催化的. 图 3 显示了 $1 \mathrm{~b}$ 浓度小于底物 PNPP 浓度时观察到的突现 (burst) 动力学过程. 按 照 Bender 稳态处理方法 ${ }^{[8]}$, 可求出 $1 \mathrm{bZn}$ 对 PNPP 水解的去酰化常数为 $6.6 \times 10^{-3} \mathrm{~s}^{-1}$ (条 件如图 3 中 4). 而对于 $1 \mathrm{bCu}$, 因铜离子对 PNPP 的自发水解较快, 不满足稳态条件, 难以 求出去酰化常数, 但从图 3 可看出, $1 \mathrm{bCu}$ 的去 酰化速率比 $1 \mathrm{bZn}$ 大得多. 整个反应的机制可 表示为:

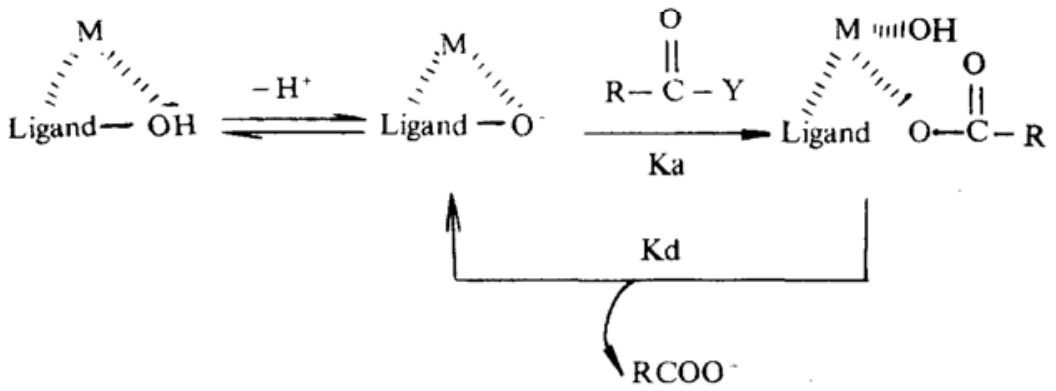

\section{3 结论}

阳离子表面活性剂型金属胶束 $1 \mathrm{a} \sim 1 \mathrm{c} Z \mathrm{n}(\mathrm{Cu})$ 对 PNPP 水解有很强的催化活性, 胶束中羟 基的活化与金属离子性质及络合物的构型有关, 季铵头的吸电子效应与空间位阻对其络合物 的形成、反应活性具有显著影响. 
致谢感谢中国科学院生物物理研究所生物大分子国家重点实验室舒占永博士在停流 实验中的热忱帮助。

\section{参考文献}

1 Scrimin P, Tecilla P, Tonellato U. Cationic metallovesicles: catalysis of the cleavage of p-nitrophenyl picolinate and control of copper(II) permeation. J Am Chem Soc, 1992, 114(13): 5 086 5 092

2 Ogino K, Yoshida T, Yamamoto $\mathrm{H}$ et al . Location of catalytic head group of 2-hydroxymethylimidazole $\mathrm{Cu}^{2+}$ complex on the micellar surface of anionic SDS in the hydrolysis of p-nitrophenyl picolinate. Chem Lett, 1992, 247(7): $1197 \sim 1200$

3 Tagaki W, Ogino K. Micellar models of zinc enzymes. Top Curr Chem, 1985, 128: 143 174

4 Ogino K, Kashihara N, Ueda T et al. Hydrolytic metalloenzyme models. Metal ion dependent site selective acylation of hydroxyl groups of bis-imidazole ligands catalyzed by $\mathrm{Zn}^{2+}$ and $\mathrm{Cu}^{2+}$ in the reaction with p-nitrophenyl 2-pyridinecarboxylate in a cationic surfactant micelle. Bull Chem Soc Jpn, 1992, 65(2): 373 384

5 Sigman D S, Jorgensen C T. Models for metalloenzymes. The zinc(II)-catalyzed transesterification of N-( $\beta$-hydroxyethyl) ethylenediamine by p-nitrophenyl picolinate. J Am Chem Soc, 1972, 94(5) : 1724 1730

6 Fendler J H 著. 程虎民, 高月英译. 膜模拟化学. 北京:科学出版社, 1991. 8

7 Fornasier R, Milani D, Scrimin P et al. Functional micellar catalysis. Part 8. Catalysis of the hydrolysis of p-nitrophenyl picolinate by metal-chelating micelles containing copper(II) or zinc(II). J Chem Soc, Perkin Trans II, 1986, 233 237

8 Bender M L, Marshall T H. The elastase-catalyzed hydrolysis of p-nitrophenyl trimethylacetate. J Am Chem Soc, 1968, 90 : 201 207 